\title{
Deutungen: eine qualitative Studie unmittelbarer Patientenreaktionen
}

\author{
Dennis Firmansyah · Kai Mergel • Cord Benecke • Dorothea Huber • \\ Imke Grimm • Günther Klug • Miriam Henkel
}

Angenommen: 18. Februar 2021 / Online publiziert: 22. April 2021

(C) Der/die Autor(en) 2021

Zusammenfassung Klassische psychoanalytische Deutungen stießen in den letzten Jahren vermehrt auf Kritik: Demnach würden diese Deutungen der therapeutischen Beziehung schaden, Blockaden auslösen und mit einer problematischen therapeutischen Haltung einhergehen. Anhand der Tonbandaufnahmen von 35 psychoanalytischen Sitzungen im mittleren Behandlungsverlauf, die im Rahmen der Münchner Psychotherapiestudie aufgezeichnet wurden, wurden 23 klassische Deutungen in 16 Sitzungen identifiziert und die Reaktionen der Patientinnen und Patienten darauf mittels der Grounded-Theory-Methode analysiert. Die Reaktionen wurden in sieben Kategorien zusammengefasst, von denen zwei als unerwünscht gelten können (z. B. „Rückzug“ oder „Konfrontation“). Die Mehrzahl der Reaktionen der Patientinnen und Patienten auf klassische Deutungen fiel allerdings in Kategorien, die als fruchtbar angesehen werden, z. B. „kognitive Erkenntnis“ oder „emotionale Resonanz“. Demnach kann die teils heftige Kritik an klassischen Deutungen in unserer Studie nicht bestätigt werden.

D. Firmansyah · K. Mergel · Prof. Dr. phil. C. Benecke · Dr. phil. M. Henkel $(\bowtie)$ Institut für Psychologie, Universität Kassel, Holländische Str. 36-38, 34127 Kassel, Deutschland E-Mail: miriam.henkel@uni-kassel.de

Prof. Dr. phil. Dr. med. D. Huber · I. Grimm

Internationale Psychoanalytische Universität Berlin, Stromstr. 3b, 10555 Berlin, Deutschland

Dr. med. G. Klug

Klinik und Poliklinik für Psychosomatische Medizin und Psychotherapie, Klinikum rechts der Isar, Technische Universität München, Langerstr. 3, 81675 München, Deutschland 


\title{
Interpretations: a qualitative study of patients' immediate reactions
}

\begin{abstract}
Classical psychoanalytic interpretations have faced increasing criticism in recent years: according to some authors they harm the therapeutic relationship, block the patients' communicativeness, and are associated with a problematic therapeutic stance. Using a grounded theory approach, 35 audio recordings of psychoanalytic therapy sessions were analyzed, which were originally recorded as part of the $\mathrm{Mu}$ nich psychotherapy study. We identified 23 classical interpretations that occurred in 16 psychotherapeutic sessions. The patients' reactions to them were attributed to seven categories, of which two were negative (confrontations and withdrawal). The majority of patients' reactions to classical interpretations fell into categories, which were considered to be positive (e.g. cognitive insight or emotional resonance). Thus, the results of our study do not support the criticism of classical interpretations.
\end{abstract}

\section{Deutungsarbeit im Fokus}

Die Deutung gilt als zentrale psychodynamische Behandlungstechnik (Argelander 1981; Loewenstein 1968; Ungar 2015). Deutungen zielen darauf ab, bis dahin unbewusste Motive, Impulse, Affekte, Fantasien etc. ins bewusste Erleben zu bringen. Zudem können Zusammenhänge mit der Symptomatik hergestellt werden, wodurch diese einen bis dahin verborgenen Sinn erhalten kann (Argelander 1981). Eine gelungene Deutung soll Einsicht hervorrufen, deren heilende Wirkung Loewenstein (1968) sowohl auf das Erinnern vergessener Erlebnisse als auch auf die Überwindung von Widerständen zurückführt. Einsicht im psychoanalytischen Sinne ist dabei immer als emotionale Einsicht zu verstehen (Benecke 2014). Neuere empirische Studien belegen, dass Einsicht eine gewichtige Rolle bei der Wirksamkeit psychodynamischer Deutungsarbeit spielt (Høglend und Hagtvet 2019; Ulberg et al. 2017).

In den letzten Jahren kann eine rege Diskussion über die Deutungsarbeit beobachtet werden, die sich mit ihrem Stellenwert im psychoanalytischen „Werkzeugkasten“ befasst und die Funktion unterschiedlicher Deutungstypen zum Gegenstand hat (z. B. Borens 2015; Körner 2015, 2020; Plassmann, 2010, 2016a, 2016b; Steiner 1998; Ungar 2015; Will 2016, 2018, 2020). Plassmann (2010, S. 105) erweitert die Inhaltsdeutungen, die sich auf das „Was?“ psychischer Inhalte, also auf „Einfälle, Träume, Emotionen, Körperwahrnehmungen" beziehen, um die Prozessdeutungen, die sich auf das „Wie?“, also die „Umwandlungen, [und] Neuorganisation von seelischem Material" (Plassmann 2016b, S. 444) beziehen. Eine weitere wichtige Unterscheidung wurde zwischen gesättigter und ungesättigter bzw. zwischen klassischer und nichtklassischer Deutung gemacht. Während gesättigte Deutungen durch Eindeutigkeit, Unmissverständlichkeit und Bedeutungsfixierung gekennzeichnet sind, arbeiten ungesättigte Deutungen mit Metaphern und Bildern, lassen der Mehrdeutigkeit des Unbewussten Raum und dienen dazu, eine Sprache für unbewusste Inhalte zu finden (Will 2016, 2018). Die klassische oder gesättigte Deutung wurde als geschlossen, suggestiv, manipulierend, beziehungsschädigend und Assoziationsketten unterbrechend kritisiert (Ungar 2015; Will 2016; Ferro 2009; Heenen-Wolff 2016). In der vorliegenden Arbeit möchten wir anhand einer qualitativen Analyse von Patientenre- 
aktionen auf gesättigte Deutungen die geäußerte Kritik empirisch näher untersuchen. Die in der Literatur oftmals harsche Gegenüberstellung von klassischer, gesättigter Deutung und nichtklassischer, ungesättigter Deutung sollte allerdings nicht darüber hinwegtäuschen, dass auch gesättigte Deutungen immer als Verstehensangebote, als Hypothesen und Ideen vorgestellt werden sollten, die das Gegenüber zurückweisen oder aufgreifen und weiterverarbeiten kann (Benecke 2014). Im folgenden Abschnitt möchten wir der geäußerten Kritik an Deutungen aus theoretischer Sicht nachgehen, welche sich unseres Erachtens nach drei Themen zuordnen lässt: die potenzielle Schädlichkeit der gesättigten Deutung für die therapeutische Beziehung und die Mitteilsamkeit der Patientinnen und Patienten sowie die dahinter liegende therapeutische Haltung.

\section{Der Einfluss klassischer Deutungen auf die therapeutische Beziehung}

So bescheinigt der italienische Psychoanalytiker Ferro (2008, S. 221) der klassischen oder gesättigten Deutung Folgendes: „Die saturierte Deutung, die exzessiv entschlüsselt, wird als etwas sehr Gewalttätiges empfunden, als etwas, das verletzt und nicht nur nichts entstehen lässt, sondern letztlich sogar etwas tötet (das Vertrauen), das gerade im Entstehen ist“". Ferro (2009) präsentiert einige Beispiele, wie Patientinnen und Patienten mit gewaltvollen Metaphern auf gesättigte Deutungen reagieren. Körner $(2015,2020)$ geht ebenfalls auf die Wirkung der klassischen Deutungen auf die therapeutische Beziehung ein: Unter klassischen Deutungen lassen sich quasikausale Erklärungen zusammenfassen, also Deutungen, die Ursachen und Ursprünge von Verhaltens- und Erlebensmustern benennen. Quasikausale Erklärungen sind beispielsweise rekonstruktive und genetische Deutungen. Sie können nach Körner (2015) in der therapeutischen Beziehung die Autorität des Analytikers, der Analytikerin festigen, wobei Patientinnen und Patienten sich durch sie „unwissend“ fühlen können. Das ist sicher kein erwünschter Effekt, zielen die psychodynamischen Therapien doch darauf ab, Einsicht, nicht Unwissenheit in den Patientinnen und Patienten hervorzubringen. Die klassische Deutung stößt auch bei Rudolf (2012, S. 366) auf Kritik, dessen Therapieansatz einen ,weitgehenden Verzicht auf Deutungen“" beinhaltet. Der Vertreter der strukturbezogenen Psychotherapie weist auf die mögliche negative Wirkung klassischer Deutungen gerade bei strukturell beeinträchtigten Patientinnen und Patienten hin. Sie können, so Rudolf, ,kognitiv sowie emotional überfordern und verwirren“. Doch wie sieht es mit empirischen Erkenntnissen dazu aus?

Deutungsarbeit kann (Petraglia et al. 2015; Gumz 2018), muss aber nicht beziehungsschädigend sein (Levy et al. 2015). Sie ist in ihrer positiven Wirkung auf die therapeutische Beziehung an die Bedingungen geknüpft, eigene Anteile am Beziehungsgeschehen einzuräumen (Gumz 2018) und in der Ausgestaltung möglichst präzise an den unbewussten Konflikten und Wünschen der Patientinnen und Patienten zu bleiben (Crits-Christoph et al. 1988, 1993). Leibovich et al. (2020) kommen auf der Grundlage empirischer Fallstudien zu dem Ergebnis, dass Deutungen die therapeutische Allianz dann stärken, wenn sie an die Stärken der Patientinnen und Patienten anknüpfen und empowernd sowie wachstumsförderlich formuliert werden. Barber et al. (2013) weisen auf empirische Erkenntnisse hin, nach denen Deutungs- 
arbeit vermehrt als feindselig wahrgenommen werden und Brüche in der therapeutischen Allianz auslösen kann. Die widersprüchlichen Ergebnisse könnten sich aufklären, wenn die Studien stärker zwischen einerseits den klassischen Deutungen (in Körners Worten „quasikausale Erklärungen“ oder bei Will und Ferro „gesättigte Deutungen" genannt) und den ungesättigten Deutungen (Will, Ferro) oder Interpretationen (Körner) differenzieren würden. Bisher scheint die Forschung sich eher auf eine Differenzierung nach dem Inhalt der Deutungen, insbesondere auf Übertragungsdeutungen (Høglend et al. 2008; Kernberg et al. 2008; Nissen-Lie et al. 2020; Ulberg et al. 2014) und Widerstandsdeutungen (Bhatia et al. 2016; Olson et al. 2011; Perry und Bond 2017; Petraglia et al. 2015), zu fokussieren.

Im nächsten Abschnitt möchten wir auf eine weitere Form der Kritik eingehen, der zufolge klassische Deutungen die Bereitschaft der Patientinnen und Patienten hemmt, sich zu öffnen und sich mitzuteilen.

\section{Die Wirkung klassischer Deutungen auf die Mitteilsamkeit}

Ferro (2009, S. 16, 17) räumt ein, dass eine Behandlung neuen Antrieb erfahren könne, wenn ,,durch eine Deutung ganz entschieden und radikal eine Zäsur“ gesetzt werde. Diese ermögliche es, hinter den manifesten Äußerungen der Patientinnen und Patienten ,einen ganz anderen, tieferen Sinn aufzudecken“. Im Hinblick auf die gesättigten Deutungen überwiegen aber eindeutig die Warnungen - gerade im Hinblick auf das Fließen des Unbewussten in den Patientinnen und Patienten: Eine ,starke und unmissverständliche Deutung“ blockiere das Erkennen (Ferro 2009, S. 18). Ferro warnt vor der Gefahr ,einer Flaschenhals-Deutung, welche die Gedankenentwicklung blockiert“" (Ferro 2009, S. 195). Die gesättigte Deutung verschließe die Möglichkeit, Assoziationen und neue Szenarien in den Patientinnen und Patienten zu veranlassen. Eine Sorge, die auch die Psychoanalytikerin Heenen-Wolff (2016, S. 55) teilt, der zufolge Interventionen generell „die unerwünschte Begleiterscheinung [haben], das freie Sprechen oder Assoziieren des Patienten zu stören“. Die Nebenwirkung, die Gedankengänge der Patientinnen und Patienten durch eine Intervention, selbst wenn sie noch so empathisch zu sein scheint, einzuschränken, kann somit den Zugang zum Unbewussten versperren. Unter Rückgriff auf ein Zitat von Ferenczi beklagt sie den „Deutungsfanatismus“ als Kinderkrankheit in der Psychoanalyse und plädiert für einen sparsamen, zurückhaltenden Umgang mit Deutungen.

Wir haben gesehen, dass gesättigte Deutungen als potenziell beziehungsschädlich und im Hinblick auf das Unbewusste als blockierend kritisiert wurden. Die Kritik ist aber keine absolute, weil mehrere Autoren einräumen, dass es Situationen geben kann, in denen es erstrebenswert ist, eine Bedeutung zu fixieren und so klar und unmissverständlich wie möglich zu deuten. Temporäre Brüche in der therapeutischen Beziehung können gleichfalls nicht nur als negativ bewertet werden, da die Erfahrung mit der „Rupture-repair“-Methode (3RS, Eubanks et al. 2015) gezeigt hat, dass Prozesse vollkommen ohne Brüche nicht produktiv sind, und dass es auf längere Sicht beziehungsförderlich sein kann, Brüche in der Beziehung mit klärender Absicht anzusprechen. Sogenannte Rupture-repair-Episoden können demnach innerhalb einer therapeutischen Beziehung bedeutsame Veränderungsprozesse 
in Gang setzen. Die Reparierbarkeit von Brüchen in der therapeutischen Beziehung, die infolge einer Deutung auftreten, könnte davon abhängen, mit welcher Haltung gedeutet wird, was zu unserem dritten Thema in Bezug auf die Kritik an klassischen Deutungen überleitet.

\section{Die therapeutische Haltung im Wandel der Deutungsarbeit}

Insofern ist neben den technischen Aspekten wie dem Sättigungsgrad, dem Maß der Abgeschlossenheit einer Deutung oder dem Anteil metaphorischer Komponenten in den Interventionen auch die Haltung angesprochen, mit der Analytikerinnen und Analytiker in den Kontakt mit ihren Patientinnen und Patienten hineingehen. Ferros Gegenentwurf zu gesättigten Deutungen sind transformative Konarrationen. Ein Kernbestandteil dieser ist laut Ferro die dialogische Haltung, die einschließt, dass weder Analytikerinnen und Analytiker noch Patientinnen und Patienten ,im Besitz einer vorab feststehenden Wahrheit“" (Ferro 2009, S. 10) sind. Borens (2015, S. 51) würde Ferro vermutlich zustimmen, empfiehlt er mit Lacan den Analytikerinnen und Analytikern doch eine Position einzunehmen, die ,unvollständig, nicht wissend und schon gar nicht allwissend" ist.

\section{Aktuelle Fragestellung}

Wie Analytikerinnen und Analytiker die Deutung begreifen, befindet sich im Wandel, und die klassische Form der Deutung steht in der Kritik. Empirische Studien zur Deutungsarbeit fokussieren auf Fragestellungen zu Merkmalen der Patientinnen und Patienten (Strukturniveau: Høglend et al. 2008; Einsicht: Høglend und Hagtvet 2019; Ulberg et al. 2017; Motivation, Pathologie, interpersonelle Probleme: NissenLie et al. 2020), Verbindung von Deutungen und Outcome (Høglend et al. 2008) sowie Deutung und Allianz (Petraglia et al. 2015). Sie differenzieren nach dem Bezug der Deutungen auf die Beziehung (Übertragungsdeutungen: Høglend et al. 2008; Kernberg et al. 2008; Nissen-Lie et al. 2020; Ulberg et al. 2014) bzw. auf Abwehr (Widerstandsdeutungen: Bhatia et al. 2016; Olson et al. 2011; Perry und Bond 2017; Petraglia et al. 2015). Unterscheidungen zwischen dem Sättigungsgrad von Deutungen wurden empirisch noch nicht getroffen.

Wir wollen mit dieser Studie dazu beitragen, erste Antworten auf die Frage zu geben, wie die Kritik an den klassischen Deutungen zu bewerten ist. Da, wie oben beschrieben, diese Kritik häufig in Bezug zu den Patientenreaktionen (zerstört Vertrauen, hemmt Mitteilsamkeit, Patientinnen und Patienten fühlen sich unwissend oder bedroht) formuliert wurde, wollen wir genau diese Patientenreaktionen auf klassische bzw. gesättigte Deutungen untersuchen. Dafür wählen wir die GroundedTheory-Methodologie, wie der folgende Abschnitt darlegt. 


\section{Methode}

Das, was wir klassische Deutungen nennen, wurde von verschiedenen Autorinnen und Autoren unterschiedlich genannt, wie wir im Theorieteil gesehen haben. Auf der Grundlage der Literatur wurde ein Kriterienkatalog entwickelt, anhand dessen diese klassischen bzw. gesättigten Deutungen identifiziert wurden. Er beinhaltet drei Annahmen: Deutungen benennen eine Ursache für Erleben oder Verhalten (1), sind im Inhalt klar und unmissverständlich formuliert (2) und beziehen sich auf etwas, das den Patientinnen und Patienten nicht schon bewusst ist (3).

Unser Vorgehen bestand darin, diejenigen Interventionen herauszufiltern, auf die diese drei Kriterien zutreffen. Wenn die beiden Rater (DF, KM) die Kriterien nach einer konsensualen Diskussion als gegeben ansahen, wurde die Intervention als klassische Deutung gewertet und transkribiert. Die Transkription endete, wenn die unmittelbare Patientenreaktion auf die Deutung abgeschlossen war und ein neuer Sinnabschnitt begann. Eine an der Datenauswertung unbeteiligte praktisch tätige klinische Therapeutin in fortgeschrittener psychoanalytischer Ausbildung (MH) beurteilte im Anschluss diejenigen Deutungen, die von den Ratern als klassische Deutungen gewertet wurden, und bestätigte oder verneinte, dass es sich um klassische Deutungen handelt. Nur die Transkripte der auf diese Weise extern validierten klassischen Deutungen und die dazugehörigen Reaktionen gehen in die Auswertung ein. Sie sind die Fundstellen und damit die Grundlage für das so genannte offene Codieren (Strübing 2018). Das offene Codieren ist Bestandteil der Grounded-Theory-Methode (Mey und Mruck 2011), die im Rahmen der psychologischen, qualitativen Sozialforschung als etabliert gelten kann (Frommer und Lange 2010). Die Reaktionen der Patienten auf die Deutungen wurden transkribiert und mehrere Zeilen unter offenen Codes zusammengefasst, um das Material zu verdichten. Als Beispiel kann eine Stelle im Transkript dienen, an der eine Patientin wie folgt auf die Deutung reagiert: „Ja, aber, es ist, irgendwie, für mich vielleicht. ... So momentan, also, Sie wissen ja“. Diese beiden Zeilen wurden unter dem offenen Code ,sucht nach Worten“ zusammengefasst. Insgesamt wurden 230 offene Codes vergeben, um das Material weiter zu verdichten und eine intensive Auseinandersetzung mit den Reaktionen auf Deutungen anzuregen. Die auf diese Weise verdichteten Informationen zu den Deutungsreaktionen der Patientinnen und Patienten gingen dann in den Prozess des axialen Codierens ein. Während der Prozess des offenen Codierens eine sehr engmaschige Orientierung an dem Wortlaut der Patientenreaktionen erforderte, schufen die 230 offenen Codes gleichzeitig die Grundlage, die Informationen weiter zu verdichten: Im Rahmen des axialen Codierens wurden auf der Grundlage der offenen Codes Deutung-Reaktion-Sequenzen formuliert. Das axiale Codieren fasste nun nicht mehr mehrere Zeilen eines Transkripts zusammen, sondern das vollständige Transkript einer Reaktion. Einige solcher Deutung-ReaktionSequenzen veranschaulicht Tab. 1.

Es konnten insgesamt 23 solcher Deutung-Reaktion-Sequenzen zusammengetragen werden. Die verschiedenen Deutung-Reaktion-Sequenzen wurden dann bereits bestehenden Kategorien zugeordnet, die sich aus der theoretischen Diskussion weiter oben ergeben. 
Tab. 1 Deutungs-Reaktions-Sequenzen

\begin{tabular}{|c|c|c|}
\hline Fall & Deutung & Reaktion \\
\hline Frau B & $\begin{array}{l}\text { Der Therapeut deutet einen un- } \\
\text { bewussten Konflikt der Patien- } \\
\text { tin, der in mehreren Situationen } \\
\text { durchscheint. Er besteht darin, } \\
\text { einerseits zwar den eigenen } \\
\text { Fähigkeiten und der eigenen } \\
\text { Bewältigungskompetenzen zu } \\
\text { vertrauen, sich gleichzeitig aber } \\
\text { täuschen zu lassen. }\end{array}$ & $\begin{array}{l}\text { Die Patientin stimmt der Deutung zu (,,ja, das ist, ja“) } \\
\text { und schweigt anschließend für } 90 \mathrm{~s} \text {. In dieser Zeit scheint } \\
\text { sie intensiv zu verarbeiten und sucht nach den passenden } \\
\text { Worten, bevor sie negative Emotionen wie Panik und Ver- } \\
\text { sagensangst benennt. Ein Schweigen von } 26 \text { s scheint ihr } \\
\text { negatives emotionales Erleben weiter zu vertiefen, sie er- } \\
\text { kennt einerseits flüchtig ihre Potenziale an, bevor sie ihre } \\
\text { Reaktion mit einem Bild abschließt, das ihre Verletzlich- } \\
\text { keit illustriert: ,als ob ich ausgeliefert wäre an die Welt.“ }\end{array}$ \\
\hline Frau G & $\begin{array}{l}\text { Die Therapeutin deutet die } \\
\text { Sorge der Patientin um ihren } \\
\text { Körper als verschobene Angst- } \\
\text { und Schamgefühle. Die Deu- } \\
\text { tung bleibt aber abstrakt, wie } \\
\text { die Therapeutin am Ende der } \\
\text { Deutung selbst feststellt: „Das } \\
\text { war jetzt sehr allgemein.“ }\end{array}$ & $\begin{array}{l}\text { Die Patientin reagiert wiederholt mit Unverständnis, bevor } \\
\text { sie nachfragt. Sie schweigt und schließt ihre Reaktion } \\
\text { mit einem Rückzug ab, der darin besteht, der Therapeutin } \\
\text { - von längerem Schweigen begleitet - zuzustimmen, ohne } \\
\text { neue Assoziationen oder emotionale Resonanz: „Ja, ich } \\
\text { find' alles gut.“ }\end{array}$ \\
\hline Frau $\mathrm{H}$ & $\begin{array}{l}\text { Der Therapeut deutet den Kopf- } \\
\text { schmerz der Patientin als Re- } \\
\text { sultat eines inneren Zerwürfnis- } \\
\text { ses. }\end{array}$ & $\begin{array}{l}\text { Die Patientin ringt nach Worten, schweigt dazwischen kurz } \\
\text { und schildert Gefühle von Unwissenheit und Rastlosigkeit. } \\
\text { Sie beschreibt daraufhin die Therapie als Zumutung. Sie } \\
\text { „mache immer wieder alles mit“, äußert Zweifel an der } \\
\text { Therapie, ein „Hin-und-her.“ Warum sie die Therapie } \\
\text { fortsetzt, findet sie „echt schwierig zu sagen.“" }\end{array}$ \\
\hline
\end{tabular}

\section{Theoriegeleitete Kategorien}

Die sieben Kategorien, die wir aus der Theorie abgeleitet haben, führt Tab. 2 auf. Die Kategorien „Konfrontation“ (1) und „Rückzug“ (2) übernehmen die beiden von Eubanks et al. $(2015,2019)$ in der Rupture-repair-Methode herausgearbeiteten Arten von Brüchen. Neue Assoziationen, fließendes Material (3) als Kategorie einzuführen, beruht auf dem in einem Artikel von Thomä und Houben (1967) diskutierten Patientenreaktionskatalog von Isaacs (1939) und auf der Subskala der Transference Work Scale (Ulberg et al. 2014). Eine unmittelbare Reaktion auf Deutungen könnte eben sowohl zu einem Blockieren der Patientinnen und Patienten führen (,,BruchRückzug“) als auch zu einer Belebung (Will 2018). Wie oben bereits beschrieben, zielen Deutungen auf Einsicht ab, welche sowohl emotionale Resonanz (4) als auch kognitive Erkenntnisse (5) beinhaltet (z.B. Benecke 2014). Eine weitere Kategorie bilden haltungsbezogene Reaktionen (6) der Patientinnen und Patienten, etwa, indem sie auf die Haltung der Analytikerin, des Analytikers bei der Deutung eingehen. Die letzte Kategorie „neue Beziehungswirklichkeit“ (7) lehnt sich an Will (2018) und Körner (2020, S. 61) an, die beschreiben, dass durch Deutungen veränderte Vorstellungen von der therapeutischen Beziehung hervorgerufen werden können.

\section{Datenauswahl: Münchner Psychotherapiestudie}

Die Tonbandaufnahmen von psychoanalytischen Therapiesitzungen aus der mittleren Behandlungsphase wurden untersucht. Wir haben Behandlungen der mittleren Behandlungsphase ausgewählt, weil wir uns dort eine größere Dichte klassischer 
Tab. 2 A-priori theoriegeleitete Kategorien von Deutungsreaktionen

\begin{tabular}{|c|c|c|}
\hline Kategorie & & Beobachtungsrelevant \\
\hline 1 & Konfrontation & $\begin{array}{l}\text { Beschwerden oder Bedenken hinsichtlich der Therapie, der Aktivitäten in } \\
\text { der Therapie, des Therapiefortschritts, der Parameter der Therapie; Zurück- } \\
\text { weisungen der therapeutischen Intervention ohne Haltungsbezug; Versuche, } \\
\text { die Therapeutinnen und Therapeuten unter Druck zu setzen oder sie zu } \\
\text { kontrollieren }\end{array}$ \\
\hline 2 & Rückzug & $\begin{array}{l}\text { Das Thema wechseln, auf vermeidende Art Storytelling betreiben, Inhal- } \\
\text { te oder Affekte abspalten, minimale Antworten geben, unterwürfig oder } \\
\text { beschwichtigend reagieren, sich selbst kritisieren, in Hoffnungslosigkeit } \\
\text { verfallen, abstrakte Kommunikation pflegen oder einen manifesten affekti- } \\
\text { ven Zustand leugnen }\end{array}$ \\
\hline 3 & $\begin{array}{l}\text { Assoziationen, } \\
\text { fließendes } \\
\text { Material }\end{array}$ & $\begin{array}{l}\text { Träume, Erinnerungen, reale Lebensschwierigkeiten, neue Assoziationen, } \\
\text { oder Reflektionen werden berichtet }\end{array}$ \\
\hline 4 & $\begin{array}{l}\text { Emotionale } \\
\text { Resonanz }\end{array}$ & $\begin{array}{l}\text { Affektbelebung (Patientinnen und Patienten reagieren belebt, vitalisiert), } \\
\text { Affektmutation (Affekte verändern sich), emotionales Erleben, neue Affek- } \\
\text { te, Affektentstehung }\end{array}$ \\
\hline 5 & $\begin{array}{l}\text { Kognitive } \\
\text { Erkenntnis }\end{array}$ & $\begin{array}{l}\text { Patientinnen und Patienten beginnen, unbewusste Konflikte zu erkennen, } \\
\text { oder verstehen ihr Ausmaß besser }\end{array}$ \\
\hline 6 & $\begin{array}{l}\text { Haltungs- } \\
\text { bezogene } \\
\text { Reaktion }\end{array}$ & Patientinnen und Patienten nehmen Bezug auf die therapeutische Haltung \\
\hline 7 & $\begin{array}{l}\text { Neue Bezie- } \\
\text { hungswirk- } \\
\text { lichkeit }\end{array}$ & $\begin{array}{l}\text { Therapeutinnen und Therapeuten werden weniger als gefährlich und mehr } \\
\text { als hilfreich beschrieben }\end{array}$ \\
\hline
\end{tabular}

Deutungen erwarteten als in anderen Behandlungsphasen (Zimmermann et al. 2015). Die untersuchten Sitzungen stammen aus der Münchner Psychotherapiestudie (MPS; Huber et al. 2012; Huber und Klug 2016). Die Studie vergleicht tiefenpsychologisch fundierte, analytische und verhaltenstherapeutische Psychotherapie bei depressiven Störungen. Zu Ergebnissen der MPS siehe beispielsweise Huber et al. (2012), Zimmermann et al. (2015) und Huber et al. (2017). In diesen Artikeln ist auch die Gesamtstichprobe genau beschrieben.

In der MPS gab es 35 analytische Behandlungen, von denen jedoch acht wegen unzureichender Tonqualität der Aufnahmen nicht in die Auswertung aufgenommen werden konnten. In acht der verbleibenden 27 mittleren Behandlungsstunden konnten keine gesättigten Deutungen gefunden werden. In drei weiteren gab es zwar gesättigte Deutungen, diese vollzogen sich jedoch über eine längere Zeit im Verlauf der Sitzung, sodass die unmittelbaren Patientenreaktionen nicht genau genug eruiert werden konnten. In den verbleibenden 16 Sitzungen wurden insgesamt 23 gesättigte Deutungen gefunden. Die Stichprobe der zugehörigen 16 Patientinnen und Patienten lässt sich wie folgt charakterisieren: Zehn waren weiblich und sechs männlich. Das mittlere Alter betrug 31,8 Jahre (SD \pm 6,3 Jahre) und schwankte zwischen 23 und 47 Jahren. Fünf der elf Patienten wiesen eine komorbide Persönlichkeitsstörung auf. Der Gesamtwert des Beck-Depressions-Inventars betrug im Mittel 26,8 $(\mathrm{SD} \pm 7,8)$, der Global Severity Index 1,3 (SD \pm 0,5) und der Gesamtwert des Inventars für interpersonelle Probleme 1,8 $(\mathrm{SD} \pm 0,5)$. Keine der beschriebenen Variablen der hier untersuchten MPS-Teilstichprobe unterschied sich signifikant von den üb- 
rigen Studienteilnehmern oder von den übrigen Teilnehmern, die zur analytischen Psychotherapie randomisiert worden waren (alle $p<0,05$ ).

\section{Ergebnisse}

Neun Deutung-Reaktion-Sequenzen wurden den unerwünschten Reaktionsweisen zugeordnet, davon zwei Konfrontationen und sieben Rückzüge. Auf die Kategorien erwünschter Reaktionsweisen entfielen in verschiedenen Kombinationen 14 Deutung-Reaktion-Sequenzen. Da sie teilweise mehreren erwünschten Reaktionsweisen zugeordnet werden konnten, addiert sich die folgende Aufzählung nicht zu ihrer Gesamtzahl. Elf Sequenzen wurden der Kategorie kognitive Erkenntnis zugeordnet. Sieben Deutung-Reaktion-Sequenzen wurden der Kategorie emotionale Resonanz zugeordnet und sechs der Kategorie neue Assoziationen. Es gab keine Reaktionsweise, die als haltungsbezogene Reaktion oder als „,neue Beziehungswirklichkeit““ $\mathrm{zu}$ interpretieren gewesen wären. Alle Deutung-Reaktion-Sequenzen konnten den theoriegeleiteten Kategorien zugeordnet werden; es war nicht notwendig, aus den Daten neue Kategorien zu schaffen. Im Folgenden wollen wir für die zwei häufigsten Kategorien der positiven Reaktionen sowie für die beiden Bruchreaktionen Beispiele zur Veranschaulichung geben.

\section{Emotionale Resonanz}

Insgesamt sieben Deutung-Reaktion-Sequenzen wurden der Kategorie ,emotionale Resonanz" zugeordnet. Frau B. (Tab. 1) reagiert auf die Deutung des Therapeuten zum Ende ihrer Antwort mit einem Schweigen von beinahe einer halben Minute. In dieser Zeit scheint sie intensive Emotionen erlebt zu haben, weil sie nach dem Schweigen ihre Angst schildert, ,in Berührung zu kommen“. Sie fühle sich, als ob sie ,,ausgeliefert wäre an die Welt". Herr C. reagiert mit Einsicht und einem AhaErlebnis auf die Deutung und zeigt dann zum Ende seiner Reaktion hin intensive Emotionen. Er reagiert erst mit Bestürzen auf die Deutung: „Ja, das ist wirklich scheiße“. Auf ein längeres Schweigen hört man ein geräuschvolles Ausatmen, das darauf schließen lässt, dass der Patient sich Luft machen muss. Daraufhin schweigt er erneut für beinahe eine Minute. Währenddessen scheint er intensiv über die Deutung nachgedacht bzw. ihr nachgespürt zu haben, weil er sie dann nochmal in eigene Worte fasst. Diese und ähnliche Reaktionen haben wir als emotionale Resonanz zusammengefasst.

\section{Kognitive Erkenntnis}

Die Deutung in eigene Worte zu fassen, sie weiterzudenken, nach ihren Konsequenzen zu fragen oder auf ihre Hintergründe einzugehen, kennzeichnet diejenigen elf Deutung-Reaktion-Sequenzen, die wir unter kognitiver Erkenntnis verbucht haben. In Form kurzer Zitate sollen diese schlaglichtartig beleuchtet werden: Etwa Frau D., die nach einer Deutung sagt, dass ihr etwas ,,jetzt total bewusst geworden“ sei. Oder Herr E., dem nach einer Traumdeutung klar wird, dass er seiner Tochter nicht im- 
mer nur Gutes will: ,[es] ist eben oft auch so, dass ich sie irgendwie unbewusst angreife“. Frau F. reagiert auf die Deutung ihres schlechten Gewissens einsichtig, indem sie die Ursache bestätigt und in eigene Worte fasst: „,vielleicht habe ich mich dann deswegen noch ein bisschen schlechter gefühlt."

\section{Rückzug}

Innerhalb der Rückzugskategorie finden sich Deutung-Reaktion-Sequenzen, die oftmals auf den ersten Blick den Deutungen zustimmen, sie aber nicht verwenden, um ihre Erlebens- und Verhaltensweisen aufzuschlüsseln. Im Falle der Frau G. (Tab. 1) wird dies besonders deutlich:

Ein Blick in das Transkript der Sitzung zeigt, dass die Patientin gleich dreimal ihre Zustimmung verbalisiert: „Ja, das ist doch gut.“, ,Ja, ich find' alles gut.“ und zum Ende hin „Das ist doch eigentlich gut“. Es drängt sich der Eindruck auf, dass die Patientin mit der Deutung nichts anfangen kann und ihre Zustimmung als minimale Antwort gemeint ist, die die Therapeutin beschwichtigen soll, aber nicht dazu führt, dass unbewusste Inhalte bewusst werden.

\section{Konfrontation}

Im Falle der Reaktion von Frau H. (Tab. 1) ist es nicht nur nicht gelungen, unbewusste Inhalte bewusst zu machen. Die Deutung führt hier gar zu einem Konfrontationsbruch, der mit dem Infragestellen der Therapie als Ganzes einhergeht.

\section{Diskussion}

Sind klassische, gesättigte Deutungen beziehungsschädlich (Ferro 2008, 2009)? Lösen Sie bei Patientinnen und Patienten aus, sich zurückzuziehen, zu blockieren (Heenen-Wolff 2016; Ferro 2009) oder in eine Konfrontation mit den Analytikern, Analytikerinnen zu gehen? Oder unterstützen sie die Heilung von Patientinnen und Patienten, indem sie Einsicht ermöglichen (Argelander 1981; Høglend und Hagtvet 2019; Ulberg et al. 2017), den Impuls für neue Assoziationen geben und eine neue Beziehungswirklichkeit herstellen (Will 2018; Körner 2020)? Gemäß der zum Teil sehr drastisch formulierten Kritik (z. B. Ferro) an klassischen bzw. gesättigten Deutungen wären überwiegend negative Reaktionen von Patientinnen und Patienten auf diese Form der Deutung zu erwarten gewesen.

Anhand der Tonbandaufnahmen von 27 psychoanalytischen Sitzungen im mittleren Behandlungsverlauf, die im Rahmen der MPS (Huber und Klug 2016) aufgezeichnet wurden, wurden 23 gesättigte Deutungen in 16 Sitzungen identifiziert und die Reaktionen der Patientinnen und Patienten darauf mittels der Grounded-TheoryMethode analysiert. Die Reaktionen wurden in Kategorien zusammengefasst, von denen zwei als unerwünscht gelten können (,Rückzug“ oder „Konfrontation“). Entgegen der Annahme, die sich aus der Kritik an klassischen Deutungen ableitet, fiel die Mehrzahl der Reaktionen der Patientinnen und Patienten auf klassische Deutungen allerdings in Kategorien, die als fruchtbar angesehen werden, z. B. ,kognitive 
Erkenntnis“ oder „emotionale Resonanz“. Die oftmals sehr rigoros formulierte Kritik an klassischen bzw. gesättigten Deutungen kann so pauschal also keine Gültigkeit beanspruchen. Daraus ergibt sich die weiterführende Forschungsfrage, woran es liegen könnte, ob eine klassische Deutung eine eher produktive oder eher unproduktive Reaktion hervorruft. Mögliche Ansatzpunkte könnten hier, wie eingangs erwähnt, zum Beispiel die Art und Weise sein, wie eine klassische Deutung formuliert wird, also eher als Verstehensangebot/Hypothese oder eher als Tatsachenformulierung, der man kaum widersprechen kann. Weiterhin könnte auch die aktuelle Übertragungssituation in der Behandlung eine große Rolle dabei spielen, ob eine Deutung produktiv verarbeitet wird. Zudem darf nicht außer Acht gelassen werden, dass auch „Brüche“ nicht per se unproduktiv sind, sondern bei entsprechender Bearbeitung zu einem fruchtbaren Prozess beitragen können.

\section{Limitationen und Ausblick}

Eine Limitation unserer Studie besteht in der kleinen Stichprobe, wodurch einige der geäußerten Kritikpunkte, wie die Annahme von Rudolf (2012), nach der sich die unerwünschten Effekte von Deutungen gerade bei strukturell beeinträchtigten Patientinnen und Patienten zeigen, im Rahmen dieser Studie nicht untersucht werden konnten. Zudem wurden nur die unmittelbaren Reaktionen der Patientinnen und Patienten untersucht; Aussagen darüber, inwiefern die Deutungen im weiteren Verlauf der analytischen Behandlung veränderte Reaktionen hervorriefen, lassen sich dadurch nicht treffen. Es ist jedoch zu vermuten, dass ein solcher Einfluss bestehen könnte, beispielsweise, wenn eine Patientin eine Deutung anfangs ablehnt, aber im Laufe der Therapie als passend erkennt. Möglich ist, dass die in unserem Material nichtkodierten Kategorien „haltungsbezogene Reaktionen“ und „,neue Beziehungswirklichkeit" eher als langfristige Reaktionen mehrerer Deutungen auftreten und daher hier nicht leicht zu erfassen waren.

Eine Einschätzung darüber, wann eine unmittelbare Reaktion abgeschlossen ist, hat sich zudem nicht immer als eindeutig erwiesen. Zwar gab es oft klar abgrenzbare Deutungen. In manchen Sitzungen wurden Deutungen allerdings auch erst im Wortwechsel zwischen Patient und Therapeutin nach und nach entfaltet. In diesen Fällen gab es einen großen subjektiven Spielraum bei der Bestimmung, wo nun der Anfang der Deutung und der Beginn bzw. das Ende der Patientenreaktion liegt.

Die Audioaufnahmen der Therapiesitzungen wurden Ende der 1990er-Jahre und Anfang 2000 aufgezeichnet. Vor dem Hintergrund der im Wandel begriffenen Deutungsarbeit (Ungar 2015) stellt sich die Frage, ob eine Untersuchung aktueller psychoanalytischer Therapiesitzungen dieselben Ergebnisse hervorbringen würde. Einen Einfluss auf die Ergebnisse hatte zweifelsohne auch die Tatsache, dass die Mitschnitte nur den Ton, nicht aber das Bild festhielten. Ob eine Reaktion emotionale Resonanz hervorrief, ließe sich unter Berücksichtigung des mimischen Ausdrucks besser bewerten als nur anhand des Klangs der Stimme, der Modulation und des Inhalts.

Zukünftige Studien auf der Grundlage aktueller Sitzungen, an einer größeren Stichprobe und mit Patientinnen und Patienten mit unterschiedlichen Störungsbildern sind notwendig, um die Wirkung klassischer Deutungen auf Patientinnen und 
Patienten weitergehend $\mathrm{zu}$ erforschen. Außerdem könnten weitere Informationen gewonnen werden, wenn die Möglichkeit bestünde, ehemalige Patientinnen und Patienten nach Ende des therapeutischen Prozesses zu befragen, welche Einsichten ihnen bei ihrem Heilungsprozess nützlich waren. Als weitere Forschungsperspektive könnten ungesättigte Deutungen und die Reaktionen auf diese Deutungen näher betrachtet werden. So könnte man den eingangs erwähnten Annahmen der Beziehungsförderlichkeit dieser Deutungsarten empirisch näherkommen, und es könnte ein Vergleich zwischen Reaktionen auf gesättigte versus ungesättigte Deutungen erfolgen. Hierzu stellt sich jedoch zunächst die Frage, wie ungesättigte Deutungen erkannt werden könnten, denn Kriterien, wie wir sie bei den gesättigten Deutungen erstellen konnten, scheinen hier weniger klar definiert.

Funding Open Access funding enabled and organized by Projekt DEAL.

Open Access Dieser Artikel wird unter der Creative Commons Namensnennung 4.0 International Lizenz veröffentlicht, welche die Nutzung, Vervielfältigung, Bearbeitung, Verbreitung und Wiedergabe in jeglichem Medium und Format erlaubt, sofern Sie den/die ursprünglichen Autor(en) und die Quelle ordnungsgemäß nennen, einen Link zur Creative Commons Lizenz beifügen und angeben, ob Änderungen vorgenommen wurden.

Die in diesem Artikel enthaltenen Bilder und sonstiges Drittmaterial unterliegen ebenfalls der genannten Creative Commons Lizenz, sofern sich aus der Abbildungslegende nichts anderes ergibt. Sofern das betreffende Material nicht unter der genannten Creative Commons Lizenz steht und die betreffende Handlung nicht nach gesetzlichen Vorschriften erlaubt ist, ist für die oben aufgeführten Weiterverwendungen des Materials die Einwilligung des jeweiligen Rechteinhabers einzuholen.

Weitere Details zur Lizenz entnehmen Sie bitte der Lizenzinformation auf http://creativecommons.org/ licenses/by/4.0/deed.de.

Interessenkonflikt D. Firmansyah, K. Mergel, C. Benecke, D. Huber, I. Grimm, G. Klug und M. Henkel geben an, dass kein Interessenkonflikt besteht.

\section{Literatur}

Argelander H (1981) Was ist eine Deutung?: Was ist eine Deutung? Psyche 35(11):999-1005

Barber JP, Muran JC, McCarthy KS, Keefe RJ (2013) Research on psychodynamic therapies. In: Lambert MJ (Hrsg) Bergin and Garfield's handbook of psychotherapy and behavior change, 6. Aufl. John Wiley \& Sons, S 443-494

Benecke C (2014) Klinische Psychologie und Psychotherapie: Ein integratives Lehrbuch, 1. Aufl. Kohlhammer

Bhatia M, Petraglia J, de Roten Y, Banon E, Despland J-N, Drapeau M (2016) What defense mechanisms do therapists interpret in-session? Psychodyn Psychiatry 44(4):567-585. https://doi.org/10. 1521/pdps.2016.44.4.567

Borens R (2015) Soll man heute noch deuten? Psyche 69(1):47-63

Crits-Christoph P, Cooper A, Luborsky L (1988) The accuracy of therapists' interpretations and the outcome of dynamic psychotherapy. J Consult Clin Psychol 56(4):490-495. https://doi.org/10.1037/ 0022-006X.56.4.490

Crits-Christoph P, Barber J, Kurcias J (1993) The accuracy of therapists' interpretations and the development of the therapeutic alliance. Psychother Res 3:25-35. https://doi.org/10.1080/1050330931233133 3639

Eubanks CF, Muran JC, Safran JD (2015) Rupture resolution rating system (3RS): Manual. Unpublished manuscript, Mount Sinai Beth Israel Medical Center, New York. https://doi.org/10.13140/2.1.1666. 8488

Eubanks CF, Lubitz J, Muran JC, Safran JD (2019) Rupture resolution rating system (3RS): development and validation. Psychother Res 29(3):306-319. https://doi.org/10.1080/10503307.2018.1552034 
Ferro A (2008) Die Transformation. Mikrotransformationen, Makrotransformationen und Transformationen durch Narration. Forum Psychoanal (24):217-228. https://doi.org/10.1007/s00451-008-0352-2

Ferro A (2009) Psychoanalyse als Erzählkunst und Therapieform. Psychosozial

Frommer J, Lange J (2010) Psychotherapieforschung. In: Mey G, Mruck K (Hrsg) Handbuch qualitative Forschung in der Psychologie, 1. Aufl. VS, S 776-782

Gumz A (2018) Psychodynamische Interventionstechniken. In: Gumz A, Hörz-Sagstetter S (Hrsg) Psychodynamische Psychotherapie in der Praxis. Beltz

Heenen-Wolff S (2016) Über die Gründe, zu deuten, und jene, es nicht zu tun. Sprechen und Zuhören im analytischen Prozess. In: Kuttenkeuler D, Schäfer G (Hrsg) Deutungsprozesse im Wandel. Psychosozial, S 45-62

Høglend P, Hagtvet K (2019) Change mechanisms in psychotherapy: Both improved insight and improved affective awareness are necessary. J Consult Clin Psychol 87(4):332-344. https://doi.org/10.1037/ ccp0000381

Høglend P, Bøgwald K-P, Amlo S, Marble A, Ulberg R, Sjaastad MC, Sørbye Ø, Heyerdahl O, Johansson P (2008) Transference interpretations in dynamic psychotherapy: do they really yield sustained effects? Am J Psychiatry 165(6):763-771. https://doi.org/10.1176/appi.ajp.2008.07061028

Huber D, Klug G (2016) Münchner Psychotherapiestudie. Psychotherapeut 61(6):462-467. https://doi.org/ 10.1007/s00278-016-0139-7

Huber D, Zimmermann J, Henrich G, Klug G (2012) Comparison of cognitive-behaviour therapy with psychoanalytic and psychodynamic therapy for depressed patients-a three-year follow-up study. Z Psychosom Med Psychother 58(3):299-316

Huber D, Zimmermann J, Klug G (2017) Change in personality functioning during psychotherapy for depression predicts long-term outcome. Psychoanal Psychol 34(4):434-445

Isaacs S (1939) Criteria for interpretation. Int J Psychoanal 20:148-160

Kernberg OF, Yeomans FE, Clarkin JF, Levy KN (2008) Transference focused psychotherapy: overview and update. Int J Psychoanal 89(3):601-620. https://doi.org/10.1111/j.1745-8315.2008.00046.x

Körner J (2015) Die Deutung in der Psychoanalyse, 1. Aufl. Kohlhammer

Körner J (2020) Die Kunst der Deutung und die Macht der Beziehung. Vandenhoeck \& Ruprecht

Leibovich L, Wachtel PL, Nof A, Zilcha-Mano S (2020) "Take a sad song and make it better": what makes an interpretation growth facilitating for the patient? Psychotherapy 57(3):400-413. https://doi.org/10. 1037/pst0000290

Levy S, Hilsenroth M, Owen J (2015) Relationship between interpretation, alliance, and outcome in psychodynamic psychotherapy: control of therapist effects and assessment of moderator variable impact. J Nerv Ment Dis 203(6):418-424. https://doi.org/10.1097/NMD.0000000000000302

Loewenstein RM (1968) Das Problem der Deutung: Das Problem der Deutung. Psyche 22(3):187-198

Mey G, Mruck K (2011) Grounded theory reader. VS

Nissen-Lie HA, Dahl H-SJ, Høglend PA (2020) Patient factors predict therapists' emotional countertransference differently depending on whether therapists use transference work in psychodynamic therapy. Psychother Res. https://doi.org/10.1080/10503307.2020.1762947

Olson TR, Perry JC, Janzen JI, Petraglia J, Presniak MD (2011) Addressing and interpreting defense mechanisms in psychotherapy: general considerations. Psychiatry Interpers Biol Process 74(2):142-165. https://doi.org/10.1521/psyc.2011.74.2.142

Perry JC, Bond M (2017) Addressing defenses in psychotherapy to improve adaptation. Psychoanal Inqu 37(3):153-166. https://doi.org/10.1080/07351690.2017.1285185

Petraglia J, Bhatia M, De Roten Y, Despland J-N, Drapeau M (2015) An empirical investigation of defense interpretation depth, defensive functioning, and alliance strength in psychodynamic psychotherapy. Am J Psychother 69(1):1-17. https://doi.org/10.1176/appi.psychotherapy.2015.69.1.1

Plassmann R (2010) Inhaltsdeutung und Prozessdeutung. Prozessorientierte Psychotherapie. Forum Psychoanal. https://doi.org/10.1007/s00451-010-0034-8

Plassmann R (2016a) Transformationsprozesse in der Traumatherapie und die Technik der Prozessdeutung. Forum Psychoanal. https://doi.org/10.1007/s00451-015-0213-8

Plassmann R (2016b) Die Technik der Prozessdeutung. Forum Psychoanal. https://doi.org/10.1007/ s00451-016-0236-9

Rudolf G (2012) Strukturbezogene Psychotherapie. Psychotherapeut 57(4):357-372. https://doi.org/10. 1007/s00278-012-0915-y

Steiner J (1998) Probleme der psychoanalytischen Technik - Patientenzentrierte und analytikerzentrierte Deutung. In: Steiner J (Hrsg) Orte des seelischen Rückzugs. Klett-Cotta, S 191-212 
Strübing J (2018) Grounded Theory: Methodische und methodologische Grundlagen. In: Pentzold C, Bischof A, Heise N (Hrsg) Praxis Grounded Theory. Springer, Berlin Heidelberg, S 27-52 https://doi. org/10.1007/978-3-658-15999-3

Thomä H, Houben A (1967). Über die Validierung psychoanalytischer Theorien durch die Untersuchung von Deutungsaktionen. Psyche 21(9):664-692

Ulberg R, Amlo S, Høglend P (2014) Manual for transference work scale; a micro-analytical tool for therapy process analyses. BMC Psychiatry 14(1):291. https://doi.org/10.1186/s12888-014-0291-y

Ulberg R, Amlo S, Dahl H-SJ, Høglend P (2017) Does insight mediate treatment and enhance outcome? Psychoanal Inqu 37(3):140-152. https://doi.org/10.1080/07351690.2017.1285184

Ungar V (2015) Der Analytiker und sein Werkzeugkasten: Der Analytiker und sein Werkzeugkasten. Psyche 69(5):413-435

Will H (2016) Ungesättigte und gesättigte Deutungen. Psyche 70(1):2-23. https://doi.org/10.21706/ps-701-2

Will H (2018) Wie ungesättigte Deutungen entstehen: Wie ungesättigte Deutungen entstehen. Psyche 72(5):374-396

Will H (2020) Drei Dimensionen, die eine psychoanalytische Deutung ausmachen. Jahrb Psychoanal 80(80):135-158

Zimmermann J, Löffler-Stastka H, Alhabbo S, Bock A, Klug G, Huber D, Benecke C (2015) Is it all about the higher dose? Why psychoanalytic psychotherapy is an effective treatment for major depression. Clin Psychol Psychother 22:469-487

Dennis Firmansyah ist Psychologe und Psychotherapeut in Ausbildung am Horst-Eberhard Richter Institut in Gießen.

Kai Mergel ist Psychologe und Psychoanalytiker in Ausbildung (DPV). Psychotherapeutisch tätig in der Parkland-Klinik für Psychosomatik und Psychotherapie in Bad Wildungen.

Prof. Dr. phil. Cord Benecke Psychoanalytiker, Professur für Klinische Psychologie und Psychotherapie, Universität Kassel. Er ist Sprecher des Arbeitskreises Operationalisierte Psychodynamische Diagnostik (OPD) und in der wissenschaftlichen Leitung der Lindauer Psychotherapiewochen. Forschungsschwerpunkte: klinische Emotionsforschung, Psychotherapieforschung. Mitherausgeber dieser Zeitschrift.

Prof. Dr. phil. Dr. med. Dorothea Huber ist Professorin für Klinische Psychologie und Psychosomatik an der IPU Berlin. Sie ist in der wissenschaftlichen Leitung der Lindauer Psychotherapiewochen. Forschungsschwerpunkte: Psychotherapieforschung, Klinische Diagnostik und Psychometrie.

Imke Grimm ist Psychologin und wissenschaftliche Mitarbeiterin im Fachbereich Klinische Psychologie und Psychosomatik an der IPU Berlin.

Dr. med. Günther Klug ist freier Mitarbeiter an der Klinik und Poliklinik für Psychosomatische Medizin der Technischen Universität München.

Dr. phil. Miriam Henkel ist Psychologin, Psychoanalytikerin in Ausbildung (DPG) und wissenschaftliche Mitarbeiterin am Institut für Psychologie der Universität Kassel. Mitglied des Arbeitskreises Operationalisierte Psychodynamische Diagnostik (OPD). Forschungsschwerpunkte: Psychotherapie(prozess)forschung, psychodynamische Diagnostik. 